\title{
From the Liouville to the Smoluchowski equation for a colloidal solute particle in a solvent
}

\author{
Riccardo Fantoni ${ }^{1, *}$ \\ ${ }^{1}$ Università di Trieste, Dipartimento di Fisica, \\ strada Costiera 11, 34151 Grignano (Trieste), Italy
}

(Dated: September 25, 2018)

\begin{abstract}
We show how the Smoluchowski dynamics of a colloidal Brownian particle suspended in a molecular solvent can be reached starting from the microscopic Liouvillian evolution of the full classical model in the high friction limit. The integration of the solvent degrees of freedom goes through a multiple time scale perturbation expansion which removes the secular divergences. A simple dynamical Monte Carlo scheme is then proposed to solve the resulting evolution equation for the colloid solute particle. In particular we study the approach to the equilibrium Boltzmann distribution at late times and its resilience behavior at shorter times as influenced by the steepness of the external potential and the friction coefficient around their respective minima. This is very important to understand the fate of the Brownian particle's random walk and its evolution history.

PACS numbers: 05.10.Gg,05.40.Jc,05.40.Fb,05.40.Ca,36.40.Sx

Keywords: Colloidal suspension, solvent, solute, Brownian dynamics, Liouville, Fokker-Planck, Smoluchowski, Monte Carlo
\end{abstract}

* rfantoni@ts.infn.it 


\section{INTRODUCTION}

The dynamics of a many-body system can be given in terms of the time evolution of the probability phase space density of the chosen ensemble of particles. We must require that the total time derivative of the probability density vanishes so that the probability density as seen by an observer moving with a phase point along its phase space trajectory, is independent of time. Phase points of the statistical ensemble are neither created nor destroyed as time evolves.

The Liouvillian dynamics gives rise to the famous kinetic equation discovered by Boltzmann in 1872 (where the assumption of two body collisions only and of uncorrelated successive collisions are only valid at sufficiently low density) or to the exact Born-BogoliubovGreen-Kirkwood-Yvon (BBGKY) hierarchical equations.

According to BBGKY the dynamics of a single particle requires the knowledge of the two-body probability density. But if we additionally require that the particle we are looking upon has mass much larger than that of all the other particles it is possible to expand perturbatively such dynamics so to find a closed equation for just the single massive particle probability phase space density.

A colloidal suspension is made of colloidal particles immersed in a solvent. The solvent (which may be water or other liquids) is in general a molecular liquid which can be described at a microscopic level by particles much smaller and lighter than the macromolecular colloidal particle. The problem of the dynamics of the big colloidal solute particle subject to its interaction with the smaller solvent molecules in statistical equilibrium at a given absolute temperature $T$ is an interesting one. Starting from the observation in 1827 by Robert Brown of the motion of pollen grains in water and the interpretation of Albert Einstein in 1905 which was later verified experimentally by Jean Baptiste Perrin in 1908, the Brownian motion began to occupy an important role in non-equilibrium statistical physics. Even if the initial description of the colloid solute particle dynamics was an empirical one it soon became clear the microscopic origin of the Brownian motion. A complete microscopic description has to take into account the solvent in a proper way. The challenge is to be able to "remove" the degrees of freedom of the solvent in favor of the ones of the solute particle. So as to have a mesoscopic description of the dynamics of the colloid solute particle dressed by the solvent.

In the second half of last century it had been discovered [1] that it is indeed possible to 
derive the Brownian motion equations starting from the Liouvillian evolution, $\mathcal{L} \equiv i\{\mathcal{H}, \ldots\}$, with $\{\ldots\}$ the Poisson brackets and $\mathcal{H}$ the model classical Hamiltonian, of the probability density in the phase space of a model of $N$ solvent particles and the colloidal particle, $f^{[N+1]}(t)=\exp (-i \mathcal{L} t) f^{[N+1]}(0)$, to the Fokker-Planck equation [2, 3] for the dynamics of the dressed solute particle alone subject to friction. The friction coefficient $\xi$ is expressed in terms of an equilibrium average over the phase space of the solvent in the external field of the solute particle of a particular term containing the microscopic force exerted by the solvent on the colloidal particle. At the end of last century it was furthermore shown that a high friction expansion, in turn, brings [4] to the Smoluchowski equation for the colloid solute particle or its equivalent stochastic Langevin equation. $[5]$ - 8$]$.

It this work we outline a simple Monte Carlo scheme that may be used to solve the resulting Smoluchowski equation that we call Dynamical Monte Carlo (DynMC). The realistic case of a colloidal suspension of poly(methyl methacrylate) (PMMA) particles in water [9] is taken as an example. In particular we study the approach to the equilibrium Boltzmann distribution at late times and its resilience behavior at shorter times as influenced by the steepness of the external potential and the friction coefficient around their respective minima. This is very important to understand the fate of the Brownian particle's random walk and its evolution history. We test our new algorithm on the case of a harmonic one dimensional external potential for which the analytic exact solution of the Smoluchowski equation is known.

Of course a more realistic treatment of the molecular solvent would be through a quantum statistical mechanics description where $\mathcal{L} \equiv i[\hat{\mathcal{H}}, \ldots] / \hbar$, with $[\ldots]$ the commutator and $\hat{\mathcal{H}}$ the model Hamiltonian operator. Then we would have a mixed evolution where the solvent is treated quantum mechanically and the massive colloid solute particle is treated classically (see for example Ref. [10] or Ref. [11] where the full quantum mechanical treatment is considered). We leave this as an open future problem.

The paper is organized as follows: In Section【we present the reduction from the Liouville equation to the Fokker-Planck equation, in Section III we present the reduction from the Fokker-Planck equation to the Smoluchowski equation, in Section IV we present the DynMC algorithm, in Section V we carry on a computer experiment with the newly developed 
algorithm to solve for the time dependence of the mean Brownian particle position under the influence of an external harmonic potential in one spatial solution, Section VI is for final remarks.

\section{FROM THE LIOUVILLE TO THE FOKKER-PLANCK EQUATION}

In this section we will reproduce and discuss the multiple time scale perturbation expansion presented in Ref. [12] which brings from the microscopic Liouville equation for a solute particle in a solvent to its mesoscopic Fokker-Planck equation.

We consider a colloidal solute particle of diameter $\Sigma$ and mass $M$ immersed in a colloidal suspension of small solvent particles of diameter $\sigma$ and mass $m \ll M$. The Hamiltonian of the system of $N+1$ particles can thus be written as

$$
\mathcal{H}=\frac{P^{2}}{2 M}+\sum_{i=1}^{N} \frac{p_{i}^{2}}{2 m}+V_{N}\left(\mathbf{r}^{N}\right)+V_{b}\left(\mathbf{R}, \mathbf{r}^{N}\right),
$$

where $V_{N}$ is the total interaction energy of the $N$ solvent particles of coordinates $\mathbf{r}^{N}=$ $\left(\mathbf{r}_{1}, \mathbf{r}_{2}, \ldots, \mathbf{r}_{N}\right)$ and momenta $\mathbf{p}^{N}=\left(\mathbf{p}_{1}, \mathbf{p}_{2}, \ldots, \mathbf{p}_{N}\right)$, and $V_{b}$ is the potential energy of the solvent particles in the field of a Brownian solute particle placed at $\mathbf{R}$ with momentum $\mathbf{P}$. The Liouville operator splits naturally into a solvent and a Brownian terms: $\mathcal{L}=\mathcal{L}_{s}+\mathcal{L}_{b}$, with

$$
\begin{aligned}
\mathcal{L}_{s} & =-i \sum_{i=1}^{N}\left(\frac{\mathbf{p}_{i}}{m} \cdot \frac{\partial}{\partial \mathbf{r}_{i}}+\mathbf{f}_{i} \cdot \frac{\partial}{\partial \mathbf{p}_{i}}\right), \\
\mathcal{L}_{b} & =-i\left(\frac{\mathbf{P}}{M} \cdot \frac{\partial}{\partial \mathbf{R}}+\mathbf{F} \cdot \frac{\partial}{\partial \mathbf{P}}\right)
\end{aligned}
$$

where $\mathbf{f}_{i}=-\partial\left(V_{N}+V_{b}\right) / \partial \mathbf{r}_{i}$ is the force acting on the solvent particle $i$ and $\mathbf{F}=-\partial V_{b} / \partial \mathbf{R}$ is the force exerted on the solute particle by the solvent particles. The Liouville equation for the phase space probability density of the system of $N+1$ particles is therefore

$$
\frac{\partial}{\partial t} f^{[N+1]}\left(\mathbf{B}, \mathbf{b}^{N} ; t\right)=-i\left(\mathcal{L}_{s}+\mathcal{L}_{b}\right) f^{[N+1]}\left(\mathbf{B}, \mathbf{b}^{N} ; t\right),
$$

where we we use the following notation: $\mathbf{B} \equiv\{\mathbf{R}, \mathbf{P}\}$ and $\mathbf{b}^{N} \equiv\left\{\mathbf{r}^{N}, \mathbf{p}^{N}\right\}$.

We now introduce a perturbation parameter $\epsilon=\sqrt{m / M} \ll 1$ and rescale the solute particle momenta accordingly as follows: $\mathbf{P}^{\prime}=\epsilon \mathbf{P}$, so that

$$
\mathcal{L}_{b}=-i \epsilon\left(\frac{\mathbf{P}^{\prime}}{m} \cdot \frac{\partial}{\partial \mathbf{R}}+\mathbf{F} \cdot \frac{\partial}{\partial \mathbf{P}^{\prime}}\right) \equiv \epsilon \mathcal{L}_{b}^{\prime} .
$$


We want to find now the Liouville equation for the one-particle distribution function

$$
f_{b}(\mathbf{B}, t) \equiv f^{[1]}\left(\mathbf{R}, \mathbf{P}^{\prime} ; t\right)=\int f^{[N+1]}\left(\mathbf{B}, \mathbf{b}^{N} ; t\right) d \mathbf{b}^{N}
$$

Integrating then Eq. (4) over the coordinates and momenta of the solvent we find

$$
\frac{\partial}{\partial t} f_{b}(\mathbf{B}, t)=-\epsilon \frac{\mathbf{P}^{\prime}}{m} \cdot \frac{\partial}{\partial \mathbf{R}} f_{b}(\mathbf{B}, t)-\epsilon \int \mathbf{F} \cdot \frac{\partial}{\partial \mathbf{P}^{\prime}} f^{[N+1]}\left(\mathbf{B}, \mathbf{b}^{N} ; t\right) d \mathbf{b}^{N},
$$

where the term containing $\mathcal{L}_{s}$ vanishes because we assume zero net flow of probability at infinity in phase space.

The perturbation expansion in $\epsilon$ over a multiple time scale is necessary in order to extract the mesoscopic time evolution from the microscopic one due to secular divergences of the solution at sufficiently long times, irrespective of how small $\epsilon$ may be. We then introduce an auxiliary distribution function

$$
f_{\epsilon}^{[N+1]}\left(\mathbf{B}, \mathbf{b}^{N} ; t_{0}, t_{1}, t_{2}, \ldots\right)=f_{\epsilon 0}^{[N+1]}+\epsilon f_{\epsilon 1}^{[N+1]}+\epsilon^{2} f_{\epsilon 2}^{[N+1]}+\ldots
$$

which is a function of multiple time variables

$$
t_{0}=t, \quad t_{1}=\epsilon t, \quad t_{2}=\epsilon^{2} t, \quad \ldots, \quad t_{n}=\epsilon^{n} t .
$$

Eq. (8) indicates that the dependence of the distribution function on $t_{n}$ characterizes the evolution on the time scale $t \sim \epsilon^{n}$ for $n=0,1,2, \ldots$. So, the original Liouville Eq. (4) is replaced by

$$
\left(\frac{\partial}{\partial t_{0}}+\epsilon \frac{\partial}{\partial t_{1}}+\epsilon^{2} \frac{\partial}{\partial t_{2}}+\ldots\right) f_{\epsilon}^{[N+1]}=-i\left(\mathcal{L}_{s}+\epsilon \mathcal{L}_{b}^{\prime}\right) f_{\epsilon}^{[N+1]},
$$

and the Liouville equation for the Brownian motion by

$$
\left(\frac{\partial}{\partial t_{0}}+\epsilon \frac{\partial}{\partial t_{1}}+\epsilon^{2} \frac{\partial}{\partial t_{2}}+\ldots\right) f_{b \epsilon}=-\epsilon \frac{\mathbf{P}^{\prime}}{m} \cdot \frac{\partial}{\partial \mathbf{R}} f_{b \epsilon}-\epsilon \int \mathbf{F} \cdot \frac{\partial}{\partial \mathbf{P}^{\prime}} f_{\epsilon}^{[N+1]} d \mathbf{b}^{N} .
$$

Term by term integration of this equation shows that also $f_{b \epsilon}$ can be expanded in the form: $f_{b \epsilon}=f_{b 0}+\epsilon f_{b 1}+\epsilon^{2} f_{b 2}+\ldots$ The crucial difference between Eq. (8) and a conventional perturbation expansion is the fact that the auxiliary function has a physical meaning only along the so-called physical line defined by (9). We are therefore free to impose whatever boundary conditions are needed to ensure that the expansion is free of secular divergences at successive powers of $\epsilon$ at large times. The same is true of the expansion for $f_{b \epsilon}$.

We will work up to order $\epsilon^{2}$, retaining only the three time variables $t_{0}, t_{1}$, and $t_{2}$. Substituting the perturbation expansion for $f_{\epsilon}^{[N+1]}$ and $f_{b \epsilon}$ in Eq. (11) and equating coefficients of equal powers of $\epsilon$, we arrive at the following results. 
[0. ] To zeroth order in $\epsilon$ :

From Eq. (11) we find immediately

$$
\frac{\partial}{\partial t_{0}} f_{b 0}=0
$$

so that $f_{b 0}=f_{b 0}\left(\mathbf{R}, \mathbf{P}^{\prime} ; t_{1}, t_{2}, \ldots\right)$. From Eq. (10) we find

$$
\frac{\partial}{\partial t_{0}} f_{\epsilon 0}^{[N+1]}=-i \mathcal{L}_{s} f_{\epsilon 0}^{[N+1]}
$$

Since the equilibrium phase space probability density of the solvent in the presence of the Brownian solute particle at $\mathbf{R}$ satisfies the relation $\mathcal{L}_{s} f_{0}^{[N]}\left(\mathbf{b}^{N} \mid \mathbf{R}\right)=0$, the solution to Eq. (13) is simply

$$
f_{\epsilon 0}^{[N+1]}=f_{b 0}\left(\mathbf{R}, \mathbf{P}^{\prime} ; t_{1}, t_{2}\right) f_{0}^{[N]}\left(\mathbf{b}^{N} \mid \mathbf{R}\right)
$$

where

$$
\int f_{0}^{[N]}\left(\mathbf{b}^{N} \mid \mathbf{R}\right) d \mathbf{b}^{N}=1, \quad f_{0}^{[N]}\left(\mathbf{b}^{N} \mid \mathbf{R}\right) \propto e^{-\beta \mathcal{H}}, \quad \frac{\partial}{\partial \mathbf{R}} f_{0}^{[N]}=\beta \mathbf{F} f_{0}^{[N]}
$$

here $\beta=1 / k_{B} T$ with $T$ the absolute temperature. We now employ the freedom of choice of boundary condition on the auxiliary function $f_{b \epsilon}$ imposing the following initial condition: $f_{b \epsilon}\left(\mathbf{R}, \mathbf{P}^{\prime} ; t_{0}=0, t_{1}, t_{2}\right)=f_{b 0}\left(\mathbf{R}, \mathbf{P}^{\prime} ; t_{1}, t_{2}\right)$, which in turn implies that $f_{b n}\left(\mathbf{R}, \mathbf{P}^{\prime} ; t_{0}=0, t_{1}, t_{2}\right)=0$ for $n=1,2$.

[1. ] To first order in $\epsilon$ :

From Eqs. (10) and (11) we find

$$
\frac{\partial}{\partial t_{0}} f_{\epsilon 1}^{[N+1]}+\frac{\partial}{\partial t_{1}} f_{\epsilon 0}^{[N+1]}=-i \mathcal{L}_{s} f_{\epsilon 1}^{[N+1]}-i \mathcal{L}_{b}^{\prime} f_{\epsilon 0}^{[N+1]}
$$

and

$$
\frac{\partial}{\partial t_{0}} f_{b 1}+\frac{\partial}{\partial t_{1}} f_{b 0}=\int\left(-i \mathcal{L}_{b}^{\prime}\right) f_{\epsilon 0}^{[N+1]} d \mathbf{b}^{N}
$$

respectively. Eqs. (12) and (14) show that $f_{b 0}$ and $f_{\epsilon 0}^{[N+1]}$ are both independent of $t_{0}$. To avoid secular growth of $f_{b 1}$ in (17) it is necessary to impose $\partial f_{b 1} / \partial t_{0}=0$, which combined with the initial condition for $f_{b 1}$ implies that $f_{b 1}=0$ identically, and

$$
\frac{\partial}{\partial t_{1}} f_{b 0}=\int\left(-i \mathcal{L}_{b}^{\prime}\right) f_{\epsilon 0}^{[N+1]} d \mathbf{b}^{N}=-i \mathcal{L}_{b}^{\prime} f_{b 0},
$$


where in the last equality we used Eq. (14). We therefore focus on the time evolution of $f_{b 0}$. Eq. (18) shows that on the time scale $t_{1}$ the evolution of the distribution function of the Brownian particle is the same as that of a single particle in the field of the solvent. Eq. (16) can now be rearranged as follows

$$
\begin{aligned}
\left(\frac{\partial}{\partial t_{0}}+i \mathcal{L}_{s}\right) f_{\epsilon 1}^{[N+1]} & =-\left(\frac{\partial}{\partial t_{1}}+i \mathcal{L}_{b}^{\prime}\right) f_{\epsilon 0}^{[N+1]} \\
& =-\mathbf{F} \cdot\left(\frac{\beta \mathbf{P}^{\prime}}{m}+\frac{\partial}{\partial \mathbf{P}^{\prime}}\right) f_{b 0} f_{0}^{[N]}
\end{aligned}
$$

which, upon choosing the boundary condition $f_{\epsilon 1}^{[N+1]}\left(\mathbf{R}, \mathbf{P}^{\prime}, \mathbf{b}^{N} ; t_{0}=0, t_{1}, t_{2}\right)=0$, has the formal solution

$$
\begin{aligned}
& f_{\epsilon 1}^{[N+1]}\left(\mathbf{R}, \mathbf{P}^{\prime}, \mathbf{b}^{N} ; t_{0}, t_{1}, t_{2}\right)= \\
& -\int_{0}^{t_{0}} d s e^{-i \mathcal{L}_{s} s} \mathbf{F} \cdot\left(\frac{\beta \mathbf{P}^{\prime}}{m}+\frac{\partial}{\partial \mathbf{P}^{\prime}}\right) f_{b 0}\left(\mathbf{R}, \mathbf{P}^{\prime} ; t_{1}, t_{2}\right) f_{0}^{[N]}\left(\mathbf{b}^{N} \mid \mathbf{R}\right),
\end{aligned}
$$

as can be checked by direct substitution in (20).

[2. ] To second order in $\epsilon$ :

From Eq. (11) and the fact that $f_{b 1}=0$ we find

$$
\frac{\partial}{\partial t_{0}} f_{b 2}+\frac{\partial}{\partial t_{2}} f_{b 0}=\int\left(-i \mathcal{L}_{b}^{\prime}\right) f_{\epsilon 1}^{[N+1]} d \mathbf{b}^{N}
$$

Since $f_{b 0}$ is independent of $t_{0}$, secular growth is again suppressed by setting $\partial f_{b 2} / \partial t_{0}=$ 0. Substituting the solution (20) into Eq. (21) we obtain a closed equation for the evolution of $f_{b 0}\left(\mathbf{R}, \mathbf{P}^{\prime} ; t_{1}, t_{2}\right)$ :

$$
\begin{aligned}
\frac{\partial}{\partial t_{2}} f_{b 0}= & \lim _{t_{0} \rightarrow \infty} \int d \mathbf{b}^{N} f_{0}^{[N]}\left(\mathbf{b}^{N} \mid \mathbf{R}\right) i \mathcal{L}_{b}^{\prime} \\
& \times \int_{0}^{t_{0}} d s e^{-i \mathcal{L}_{s} s} \mathbf{F} \cdot\left(\frac{\beta \mathbf{P}^{\prime}}{m}+\frac{\partial}{\partial \mathbf{P}^{\prime}}\right) f_{b 0}
\end{aligned}
$$

where the limit $t_{0} \rightarrow \infty$ can be easily taken because $f_{b 0}$ is independent of $t_{0}$. Recognizing that $e^{-i \mathcal{L}_{s} s} \mathbf{F}$ is the time evolution $\mathbf{F}(-s)$ we then find

$$
\begin{aligned}
\frac{\partial}{\partial t_{2}} f_{b 0} & =\lim _{t_{0} \rightarrow \infty} \int_{0}^{t_{0}} d s\left\langle\mathcal{L}_{b}^{\prime} \mathbf{F}(-s)\right\rangle_{b} \cdot\left(\frac{\beta \mathbf{P}^{\prime}}{m}+\frac{\partial}{\partial \mathbf{P}^{\prime}}\right) f_{b 0} \\
& =\frac{1}{3} \int_{0}^{\infty} d s\langle\mathbf{F} \cdot \mathbf{F}(-s)\rangle_{b} \frac{\partial}{\partial \mathbf{P}^{\prime}} \cdot\left(\frac{\beta \mathbf{P}^{\prime}}{m}+\frac{\partial}{\partial \mathbf{P}^{\prime}}\right) f_{b 0}
\end{aligned}
$$


where $\langle\ldots\rangle_{b}=\int \ldots f_{0}^{[N]}\left(\mathbf{b}^{N} \mid \mathbf{R}\right) d \mathbf{b}^{N}$ denotes an equilibrium average over the phase space variables of the solvent particles in the external field of the solute particle.

Putting together the results obtained so far and going back to physical time and to the original momentum variable $\mathbf{P}$, we arrive finally to the Fokker-Planck (or Klein [2] and Kramers [3]) equation for $f_{b}(\mathbf{R}, \mathbf{P} ; t)$ :

$$
\begin{aligned}
\frac{\partial}{\partial t} f_{b}(\mathbf{R}, \mathbf{P} ; t) & =\left.\left(\epsilon \frac{\partial}{\partial t_{1}}+\epsilon^{2} \frac{\partial}{\partial t_{2}}\right)\right|_{t_{1}=\epsilon t, t_{2}=\epsilon^{2} t} f_{b 0}\left(\mathbf{R}, \mathbf{P} ; t_{1}, t_{2}\right) \\
& =\left[-\frac{\mathbf{P}}{M} \cdot \frac{\partial}{\partial \mathbf{R}}+\xi \frac{\partial}{\partial \mathbf{P}} \cdot\left(\mathbf{P}+\frac{M}{\beta} \frac{\partial}{\partial \mathbf{P}}\right)\right] f_{b}(\mathbf{R}, \mathbf{P} ; t)
\end{aligned}
$$

where the friction coefficient

$$
\xi=\frac{\beta}{3 M} \int\langle\mathbf{F} \cdot \mathbf{F}(-s)\rangle_{b} d s
$$

\section{FROM THE FOKKER-PLANCK TO THE SMOLUCHOWSKI EQUATION}

We now introduce a dimensionless friction $\xi_{d}=\xi \tau_{T}$ with $\tau_{T}=\Sigma / v_{T}$ a thermal time and $v_{T}=\sqrt{1 / \beta M}$ the thermal velocity. In the high friction limit one can carry on a multiple time scale perturbation expansion in the small $\epsilon_{b} \equiv 1 / \xi_{d} \ll 1$ parameter [4], which multiplies the time derivative in the Fokker-Planck equation (24), to finally reach the Smoluchowski [13] equation, governing the time evolution of the probability density in configuration space:

$$
\rho_{b}(\mathbf{R}, t)=\int f_{b}(\mathbf{R}, \mathbf{P} ; t) d \mathbf{P} .
$$

We then repeat the same multiple time scale perturbation analysis performed in the previous section replacing now $\epsilon$ with $\epsilon_{b}$. If we use the following notation

$$
\mathcal{L}_{\mathrm{FP}} \equiv \frac{\partial}{\partial \mathbf{P}} \cdot\left(\mathbf{P}+\frac{M}{\beta} \frac{\partial}{\partial \mathbf{P}}\right),
$$

the identification of different powers of $\epsilon_{b}$ in the Fokker-Planck equation gives the following relations:

$$
\begin{aligned}
\mathcal{L}_{\mathrm{FP}} f_{b 0} & =0 \\
\mathcal{L}_{\mathrm{FP}} f_{b 1} & =\tau_{T}\left[\frac{\partial}{\partial t_{0}}+\frac{\mathbf{P}}{M} \cdot \frac{\partial}{\partial \mathbf{R}}\right] f_{b 0}, \\
\mathcal{L}_{\mathrm{FP}} f_{b 2} & =\tau_{T}\left[\frac{\partial}{\partial t_{0}}+\frac{\mathbf{P}}{M} \cdot \frac{\partial}{\partial \mathbf{R}}\right] f_{b 1}+\tau_{T} \frac{\partial}{\partial t_{1}} f_{b 0} .
\end{aligned}
$$


The zeroth order equation imposes a Maxwellian distribution

$$
f_{b 0}\left(\mathbf{R}, \mathbf{P} ; t_{0}, t_{1}, \ldots\right)=\Phi\left(\mathbf{R} ; t_{0}, t_{1}, \ldots\right) e^{-\beta P^{2} / 2 M}
$$

The first order equation imposes then

$$
\mathcal{L}_{\mathrm{FP}} f_{b 1}=\tau_{T} \frac{\partial \Phi}{\partial t_{0}} e^{-\beta P^{2} / 2 M}+\tau_{T} \frac{\mathbf{P}}{M} \cdot \frac{\partial \Phi}{\partial \mathbf{R}} e^{-\beta P^{2} / 2 M} .
$$

In order to eliminate secular divergences we must require $\partial \Phi / \partial t_{0}=0$, and the first correction for the distribution function is now given by

$$
f_{b 1}\left(\mathbf{R}, \mathbf{P} ; t_{0}, t_{1}, \ldots\right)=-\tau_{T} \frac{\mathbf{P}}{M} \cdot \frac{\partial \Phi}{\partial \mathbf{R}} e^{-\beta P^{2} / 2 M}+\Psi\left(\mathbf{R} ; t_{0}, t_{1}, \ldots\right) e^{-\beta P^{2} / 2 M}
$$

The second order equation becomes

$$
\begin{aligned}
\mathcal{L}_{\mathrm{FP}} f_{b 2}= & {\left[\tau_{T} \frac{\partial \Psi}{\partial t_{0}}+\tau_{T} \frac{\partial \Phi}{\partial t_{1}}-\left(v_{T} \tau_{T}\right)^{2} \frac{\partial}{\partial \mathbf{R}} \cdot \frac{\partial \Phi}{\partial \mathbf{R}}\right] e^{-\beta P^{2} / 2 M}+\tau_{T} \frac{\mathbf{P}}{M} \cdot \frac{\partial \Psi}{\partial \mathbf{R}} e^{-\beta P^{2} / 2 M}+} \\
& {\left[\tau_{T}^{2}\left(v_{T}^{2} \frac{\partial}{\partial \mathbf{R}} \cdot \frac{\partial}{\partial \mathbf{R}}-\frac{\mathbf{P}}{M} \cdot \frac{\partial}{\partial \mathbf{R}} \frac{\mathbf{P}}{M} \cdot \frac{\partial}{\partial \mathbf{R}}\right) \Phi\right] e^{-\beta P^{2} / 2 M} }
\end{aligned}
$$

In order to remove the secular divergences as $t_{0}$ grows to infinity, we must impose $\partial \Psi / \partial t_{0}=$ 0. Moreover it is easy to show that the eigenfunctions of $\mathcal{L}_{\mathrm{FP}}$ are the functions $H_{n}$ $\exp \left(-\beta P^{2} / 2 M\right)$ where $H_{n}$ is the $n$th Hermite polynomial. Since the Maxwellian is associated with a null eigenvalue, in order to require that the integral of the right hand side of Eq. (34) in $d \mathbf{P}$ vanishes, we must impose that all terms multiplying the Maxwellian vanish, due to the orthogonality condition between the eigenfunctions. We must then have

$$
\frac{\partial \Phi}{\partial t_{1}}=v_{T}^{2} \tau_{T} \frac{\partial}{\partial \mathbf{R}} \cdot \frac{\partial \Phi}{\partial \mathbf{R}}
$$

We now can collect the results obtained so far to obtain the distribution function to order $\epsilon_{b}^{2}$ as follows

$$
f_{b}(\mathbf{R}, \mathbf{P} ; t)=\left[\Phi-\epsilon_{b} \tau_{T} \frac{\mathbf{P}}{M} \cdot \frac{\partial \Phi}{\partial \mathbf{R}}+\epsilon_{b} \Psi+O\left(\epsilon_{b}^{2}\right)\right] e^{-\beta P^{2} / 2 M}
$$

and from Eq. (26) it follows immediately

$$
\rho_{b}(\mathbf{R}, t)=\left(\frac{2 M \pi}{\beta}\right)^{3 N / 2}\left[\Phi+\epsilon_{b} \Psi+O\left(\epsilon_{b}^{2}\right)\right]
$$


Then the time evolution for $\rho_{b}$ is obtained restricting the different variables $t_{i}$ to the physical line as follows

$$
\begin{aligned}
& \frac{\partial}{\partial t} \rho_{b}(\mathbf{R}, t) \\
= & \left(\frac{\partial}{\partial t_{0}}+\epsilon_{b} \frac{\partial}{\partial t_{1}}\right)\left(\frac{2 M \pi}{\beta}\right)^{3 N / 2}\left[\Phi\left(\mathbf{R} ; t_{1}, \ldots\right)+\epsilon_{b} \Psi\left(\mathbf{R} ; t_{1}, \ldots\right)+O\left(\epsilon_{b}^{2}\right)\right]_{t_{0}=t, t_{1}=\epsilon_{b} t, \ldots} \\
= & \left.\epsilon_{b} \frac{\partial}{\partial t_{1}}\left(\frac{2 M \pi}{\beta}\right)^{3 N / 2} \Phi\left(\mathbf{R} ; t_{1}, \ldots\right)\right|_{t_{0}=t, t_{1}=\epsilon_{b} t, \ldots}+O\left(\epsilon_{b}^{2}\right) .
\end{aligned}
$$

Then using Eq. (35) we finally find the diffusion equation

$$
\frac{\partial \rho_{b}(\mathbf{R}, t)}{\partial t}=\frac{v_{T}^{2}}{\xi} \frac{\partial}{\partial \mathbf{R}} \cdot \frac{\partial \rho_{b}(\mathbf{R}, t)}{\partial \mathbf{R}}+O\left(1 / \xi_{d}^{2}\right) .
$$

If we had an external field $\mathbf{F}_{e}(\mathbf{R})=-\partial V_{e}(\mathbf{R}) / \partial \mathbf{R}$ acting on the Brownian solute particle then the initial Hamiltonian would have been

$$
\mathcal{H}=\frac{P^{2}}{2 M}+\sum_{i=1}^{N} \frac{p_{i}^{2}}{2 m}+V_{N}\left(\mathbf{r}^{N}\right)+V_{b}\left(\mathbf{R}, \mathbf{r}^{N}\right)+V_{e}(\mathbf{R}),
$$

and the Smoluchowski equation [13] becomes

$$
\frac{\partial \rho_{b}(\mathbf{R}, t)}{\partial t}=\frac{v_{T}^{2}}{\xi} \frac{\partial}{\partial \mathbf{R}} \cdot\left(\frac{\partial}{\partial \mathbf{R}}-\beta \mathbf{F}_{e}(\mathbf{R})\right) \rho_{b}(\mathbf{R}, t)
$$

which can also be written as

$$
\frac{\partial \rho_{b}(\mathbf{R}, t)}{\partial t}=\frac{v_{T}^{2}}{\xi} \frac{\partial}{\partial \mathbf{R}} e^{-\beta V_{e}(\mathbf{R})} \frac{\partial}{\partial \mathbf{R}} e^{\beta V_{e}(\mathbf{R})} \rho_{b}(\mathbf{R}, t)
$$

which shows immediately that $\rho_{b} \propto \exp \left[-\beta V_{e}(\mathbf{R})\right]$ is a stationary solution. So that calling

$$
\beta v_{T}^{2} / \xi=1 / \gamma
$$

where $\gamma$ is usually known as the mobility, we can rewrite the Smoluchowski equation (41) as

$$
\frac{\partial \rho_{b}(\mathbf{R}, t)}{\partial t}=\frac{\partial}{\partial \mathbf{R}} \cdot\left(\frac{\partial}{\partial \mathbf{R}} \frac{v_{T}^{2}}{\xi}-\frac{\mathbf{F}_{e}(\mathbf{R})}{\gamma}\right) \rho_{b}(\mathbf{R}, t),
$$

which trough Ito's calculus [14] can be proved to be equivalent to the following stochastic differential equation, the Langevin equation

$$
\gamma \dot{\mathbf{R}}=\mathbf{F}_{e}(\mathbf{R})+\sqrt{2\left(\gamma v_{T}\right)^{2} / \xi} \zeta(t)
$$

where we denote with the dot a time derivative and $\zeta$ is a white noise. In this respect Eq. (43) is considered as a case of fluctuation and dissipation theorem. It is then straightforward 
to show that for $\mathbf{F}_{e}=0$ we find $\left\langle[\mathbf{R}(t)-\mathbf{R}(0)]^{2}\right\rangle=6 D t$ with $D=v_{T}^{2} / \xi$ the diffusion constant. Equation (43) is known as the Einstein relation. The fluctuations of the particle as it undergoes its random walk are related to the drag force (or dissipation of momentum) that the particle feels as it moves through the solvent.

The Einstein relation gives an excellent way to determine Boltzmann's constant experimentally. Watch a particle perform a Brownian jitter. After time $t$, the distance traveled by the particle should be $\left\langle R^{2}\right\rangle=2 k_{B} T t / \pi \eta \Sigma$, where we have used the Stokes formula $\gamma=3 \pi \eta \Sigma$ to relate the mobility to the viscosity $\eta$. This experiment was done in 1908 by the French physicist Jean Baptiste Perrin and won him the 1926 Nobel prize.

The full form of the Langevin equation is the one with the acceleration term. Then using the relation $\mathbf{P} / M=\mathbf{V}=\mathbf{\mathbf { R }}$ we should have in a less viscous fluid the equation $M \dot{\mathbf{V}}=-\gamma \mathbf{V}+\mathbf{F}_{e}+\gamma \sqrt{2 D} \zeta$ for which we still find for $\mathbf{F}=0,\left\langle[\mathbf{R}(t)-\mathbf{R}(0)]^{2}\right\rangle=6 D t$ and $\langle\mathbf{V}(t) \cdot \mathbf{V}(t)\rangle=3 D \gamma / M$ at late times. This stochastic differential equation leads through Ito's calculus to the Fokker-Planck equation for $f_{b}(\mathbf{R}, \mathbf{P} ; t)$. This equation (24) is sometimes also called the Klein and Kramers equation and sometimes the Chandrasekhar equation.

\section{THE DYNAMICAL MONTE CARLO METHOD}

We now want to show how the Smoluchowski evolution equation can be solved by stochastic means. In nature, equilibrium distributions are generated by an evolution process. The Smoluchowski equation

$$
\frac{\partial \rho_{b}(\mathbf{R}, t)}{\partial t}=\nabla \cdot \mathbf{D}(\mathbf{R}) \cdot\left[\nabla-\beta \mathbf{F}_{e}(\mathbf{R})\right] \rho_{b}(\mathbf{R}, t)
$$

is the unique "master" equation which is:

- local in space

- goes to the Boltzmann distribution

- is Markovian

Here $\mathbf{D}(\mathbf{R})$ is, in general, a many-body tensor. In the previous section we saw that for the one-body problem it is sufficient to take it as a constant diagonal tensor $D_{i j}=\delta_{i j} D$ and $\mathbf{F}_{e}=-\nabla V_{e}$ is the external force. 
The asymptotic solution of $\rho_{b}(\mathbf{R}, t)$ will be $\rho_{0}(\mathbf{R}) \propto \exp \left[-\beta V_{e}(\mathbf{R})\right]$. It is easy to see that this distribution satisfies $d \rho_{0} / d t=0$. If we assume the process is ergodic, since it is Markovian, this must be the only solution.

Let us define Green's function: $G\left(\mathbf{R}, \mathbf{R}_{0} ; t\right)=G\left(\mathbf{R}_{0} \rightarrow \mathbf{R} ; t\right)$ is the solution to Eq. (46) with the boundary condition at zero time: $G\left(\mathbf{R}, \mathbf{R}_{0} ; 0\right)=\delta\left(\mathbf{R}-\mathbf{R}_{0}\right)$. We can prove that the Green's function satisfies detailed balance:

$$
\rho_{0}(\mathbf{R}) G\left(\mathbf{R} \rightarrow \mathbf{R}^{\prime} ; t\right)=\rho_{0}\left(\mathbf{R}^{\prime}\right) G\left(\mathbf{R}^{\prime} \rightarrow \mathbf{R} ; t\right)
$$

for any value of $t$. To do that one writes the evolution equation for the symmetrized Green's function: $\left[\rho_{0}(\mathbf{R}) / \rho_{0}\left(\mathbf{R}^{\prime}\right)\right]^{1 / 2} G\left(\mathbf{R} \rightarrow \mathbf{R}^{\prime} ; t\right)$, and sees that the right hand side of the master equation is a Hermitian operator (see Appendix $\mathrm{A}$ ) which implies that the symmetrized Green's function is symmetric in $\mathbf{R}$ and $\mathbf{R}^{\prime}$. Then the random walk must eventually have $\rho_{0}$ as its equilibrium distribution [15]. $G$ can be used for a transition probability and it will always give an acceptance probability of unity [16]. Also it gives the interesting dynamics of a viscous particles always in contact with a heat bath.

The Smoluchowski equation leads to an interesting process but we can only calculate $G$ in the short time limit. In the following we explain a general procedure for devising an algorithm of sampling $G$. Let us calculate the moments of $G$,

$$
I_{n}\left(\mathbf{R}_{0}, t\right)=\int d \mathbf{R}\left(\mathbf{R}-\mathbf{R}_{0}\right)^{n} G\left(\mathbf{R}_{0} \rightarrow \mathbf{R} ; t\right) .
$$

Take the time derivative of this equation, use the master equation on the right hand side, and integration by parts to get a simple integral over $G$ on the right hand side. We interpret this as an average $\langle\ldots\rangle$. We assume there are no absorbing surfaces of the random walks. Then,

$$
d I_{0} / d t=0
$$

This implies the normalization of $G$ is always one, so the evolution describes a process which neither creates nor destroys walks. The next moment is:

$$
d\left[I_{1}\right]_{k} / d t=\left\langle\nabla_{j} D_{k j}+D_{k j}\left[F_{e}\right]_{j} \beta\right\rangle,
$$

where we use the Einstein summation convention over the repeated indexes. Let us assume that $\mathbf{F}_{e}$ and $\nabla \mathbf{D}$ are slowly varying. Then we can replace them by the values at the initial 
point and integrate in time:

$$
\left[R_{t}\right]_{k}=\left\langle R_{k}\right\rangle=\left[R_{0}\right]_{k}+\left.t\left\langle\nabla_{j} D_{k j}(\mathbf{R})+D_{k j}(\mathbf{R})\left[F_{e}\right]_{j}(\mathbf{R}) \beta\right\rangle_{g}\right|_{\mathbf{R}=\mathbf{R}_{0}}+O\left(t^{2}\right),
$$

where $\langle\ldots\rangle_{g}$ is an average respect to the small time Green's function $G_{g}$ of Eq. (54). The equation for the second moment is:

$$
d I_{2} / d t=2\left\langle D_{i i}+\left(R-R_{0}\right)_{i}\left(\nabla_{j} D_{i j}+D_{i j}\left[F_{e}\right]_{j} \beta\right)\right\rangle
$$

Integrating in time we then find

$$
\left\langle\left(\mathbf{R}-\mathbf{R}_{0}\right)^{2}\right\rangle=\left.2\left\langle D_{i i}(\mathbf{R})\right\rangle_{g}\right|_{\mathbf{R}=\mathbf{R}_{0}} t+O\left(t^{2}\right)
$$

The solution at small time is a Gaussian distribution with the above mean and covariance, namely

$$
G_{g}\left(\mathbf{R}, \mathbf{R}_{0} ; t\right)=\frac{e^{-\left(\mathbf{R}-\mathbf{R}_{t}\right)\left(\left.4\left\langle D_{i i}(\mathbf{R})\right\rangle_{g}\right|_{\mathbf{R}=\mathbf{R}_{0}} t\right)^{-1}\left(\mathbf{R}-\mathbf{R}_{t}\right)}}{\sqrt{\left.4 \pi\left\langle D_{i i}(\mathbf{R})\right\rangle_{g}\right|_{\mathbf{R}=\mathbf{R}_{0}} t}}
$$

According to the central limit theorem, Eqs. (51) and (153) are all that is needed to simulate the random walk if the time step $t$ is sufficiently small. The effect of the external field is to push the mean position of the Brownian particle away from its current position. An outward push in directions where the external force is positive. The cage of the surrounding solvent is also present whenever the diffusion tensor is $\mathbf{R}$ dependent.

\section{A SIMPLE MONTE CARLO SIMULATION}

For some simple forms of the external potential $V_{e}$ the Smoluchowski equation admits an analytical solution. For example the one-dimensional Smoluchowski equation in empty space (which requires the probability density to vanish at spatial infinity faster than any inverse power of $R$ ) can be solved analytically for

- A linear potential $V_{e}(x)=c x$ [17],

$$
G\left(x, x_{0} ; t\right)=\frac{e^{-\frac{\left(x-x_{0}+D \beta c t\right)^{2}}{4 D t}}}{\sqrt{4 \pi D t}} .
$$


- A harmonic potential $V_{e}(x)=c x^{2} / 2$ [18],

$$
\begin{aligned}
G\left(x, x_{0} ; t\right) & =\frac{e^{-\frac{\left(x-x_{0} e^{-2 t / \tilde{t}}\right)^{2}}{2 S(t) / \beta c}}}{\sqrt{2 \pi S(t) / \beta c}} \\
S(t) & =1-e^{-4 t / \tilde{t}} \\
\tilde{t} & =2 / \beta c D .
\end{aligned}
$$

In each one of these cases a proper change of time dependent coordinates is used to reduce the Smoluchowski equation to the free diffusion equation.

Except for these simple cases in order to extract the evolution of the Green's function we must resort to a simulation. We can then use the Markovian property as follows

$$
\begin{aligned}
& G\left(\mathbf{R}, \mathbf{R}_{0} ; t-t_{0}\right)= \\
& \int G_{g}\left(\mathbf{R}, \mathbf{R}_{n} ; t-t_{n}\right) G_{g}\left(\mathbf{R}_{n}, \mathbf{R}_{n-1} ; t_{n}-t_{n-1}\right) \cdots G_{g}\left(\mathbf{R}_{1}, \mathbf{R}_{0} ; t_{1}-t_{0}\right) d \mathbf{R}_{1} d \mathbf{R}_{2} \cdots d \mathbf{R}_{n},(57)
\end{aligned}
$$

where $t_{n}-t_{n-1}=\tau$ is a small time step. At fixed $\mathbf{R}, \mathbf{R}_{0}$, and $t-t_{0}=(n+1) \tau$ we can then easily compute the Green's function $G\left(\mathbf{R}, \mathbf{R}_{0} ; t\right)$ through a Monte Carlo integration sampling the successive $G_{g}$ of Eq. (54) with the Box-Müller method (see Ref. [15] section 3.1). For small enough $\tau$ we sample $G_{g}\left(\mathbf{R}_{1}, \mathbf{R}_{0} ; \tau\right)$ at fixed $\mathbf{R}_{0}$ generating $\mathbf{R}_{1}$ then we sample $G_{g}\left(\mathbf{R}_{2}, \mathbf{R}_{1} ; \tau\right)$ generating $\mathbf{R}_{2}$ and so on for the remaining $n-2$ factors ending with the generated $\mathbf{R}_{n}$. We can then evaluate $G_{g}\left(\mathbf{R}, \mathbf{R}_{n} ; \tau\right)$ at fixed $\mathbf{R}$ on $\mathbf{R}_{n}$ with a Monte Carlo integration to find $G\left(\mathbf{R}, \mathbf{R}_{0} ;(n+1) \tau\right)$. Clearly we will have to control the convergence of the algorithm as $\tau$ gets smaller and smaller.

Eq. (57) suggests a path integral representation of the Green function solution of the Smoluchowski equation, namely

$$
G\left(\mathbf{R}, \mathbf{R}_{0} ; t-t_{0}\right) \propto \iint_{\mathbf{R}_{0}}^{\mathbf{R}} e^{-\mathcal{S}} \mathcal{D} \mathbf{R}(t),
$$

with an action

$$
\mathcal{S}=\int_{t_{0}}^{t} d t^{\prime} \frac{\sum_{k}\left[\dot{R}_{k}\left(t^{\prime}\right)-\left.\left\langle\nabla_{j} D_{k j}+D_{k j}\left[F_{e}\right]_{j} \beta\right\rangle\right|_{\mathbf{R}=\mathbf{R}\left(t^{\prime}\right)}\right]^{2}}{\left.4\left\langle D_{i i}\right\rangle\right|_{\mathbf{R}=\mathbf{R}\left(t^{\prime}\right)}},
$$

where the dot denotes a total derivative with respect to time.

If the colloidal particle is initially localized around $\overline{\mathbf{R}}_{0}=\int \mathbf{R} \rho_{b 0}(\mathbf{R}) d \mathbf{R}$ where we assume to be given $\rho_{b 0}(\mathbf{R})=\rho_{b}\left(\mathbf{R}, t_{0}\right)$ we may want to find its average position at a later time

$$
\overline{\mathbf{R}}(t)=\int \mathbf{R} G\left(\mathbf{R}, \mathbf{R}_{0} ; t-t_{0}\right) \rho_{b 0}\left(\mathbf{R}_{0}\right) d \mathbf{R} d \mathbf{R}_{0} .
$$


Relevant mesoscopic time scales are the time $\tau_{b}=1 / \xi$ over which the velocity of a Brownian particle relaxes. At room temperature, for typical values of $\xi$, we find $\tau_{b} \approx 10^{-9} \mathrm{~s}$. Taking $\Sigma \approx 1 \mu m$ and $M \approx 10^{-21} \mathrm{~kg}[9,19]$ at room temperature $1 / \beta \approx 10^{-21} \mathrm{~J}$ we have $v_{T} \approx 1 \mathrm{~m} / \mathrm{s}$ and $\tau_{T} \approx 10^{-6} \mathrm{~s}$. Another relevant time scale is the time required for an isolated Brownian particle to diffuse over a distance equal to its diameter $\tau_{c}=\Sigma^{2} / D=\xi \beta M \Sigma^{2} \approx$ $10^{-3} \mathrm{~s}>\tau_{T}>\tau_{b}$. On the microscopic level the shortest time scale is the Enskog mean collision time $\tau_{E}=1 / \Gamma_{E}=\sqrt{\beta m / \pi} / 4 \rho_{s} \sigma^{2} g(\sigma)$ where $g(r)$ (see Ref. [12] section 2.5) is the radial distribution function of the solvent. For a solvent such as water $\sigma=\Sigma / 10^{4}$, and $\tau_{E} \approx 10^{-12} \mathrm{~s}$. We already know that for $t \gg \tau_{E}$ the Smoluchowski solution tends to the equilibrium Boltzmann distribution which remains then stable on a large subsequent time scale. For example, for the one dimensional harmonic potential with an initial gaussian distribution centered on $x_{0}$ we find

$$
\bar{x}(t)=\frac{\sqrt{2} x_{0} e^{-\beta c D t}}{\sqrt{\left(1-e^{-2 \beta c D t}\right)[1+\operatorname{coth}(\beta c D t)]}},
$$

showing that the relaxation time is proportional to $1 / \beta c D$. In Fig. 1 we show a DynMC simulation of this case for $\beta c D=1 \mathrm{~s}^{-1}, x_{0}=2 \mathrm{~m}$, and fixed $\tau=0.001 \mathrm{~s}$. Clearly at long

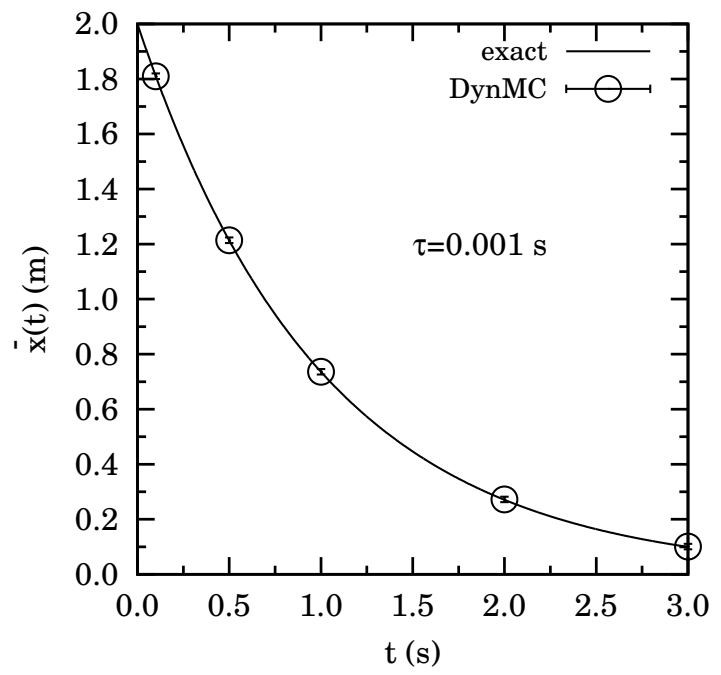

FIG. 1. DynMC simulation for the one dimensional harmonic potential with an initial gaussian distribution centered on $x_{0}=2 \mathrm{~m}$ with $\beta c D=1 \mathrm{~s}^{-1}$ and $\tau=0.001 \mathrm{~s}$. In this case $\left\langle F_{e}(x)\right\rangle_{g}=$ $-c x /(1+\beta c D \tau)$. The continuous line is the exact analytical solution of Eq. (61) and the symbols are the results of the simulation. 
enough times the solute particle will reach the equilibrium average position

$$
\overline{\mathbf{R}}_{e}=\frac{\int \mathbf{R} e^{-\beta V_{e}(\mathbf{R})} d \mathbf{R}}{\int e^{-\beta V_{e}(\mathbf{R})} d \mathbf{R}}
$$

The natural time scale over which the Smoluchowski evolution approach equilibrium is $\tau_{b}$. What may be interesting to observe is whether a proper control of the external fields allows the approach to equilibrium to happens more rapidly, thus indicating a "persistence" of the Boltzmann distribution since early times. Clearly if $V_{e}(\mathbf{R})=V_{e}(R)$ one must end up in $\overline{\mathbf{R}}_{e}=0$, by symmetry. For a single Brownian particle Eq. (51) becomes

$$
\mathbf{R}_{\tau} \simeq \mathbf{R}_{0}+\left.\tau D \beta\left\langle\mathbf{F}_{e}\right\rangle_{g}\right|_{\mathbf{R}=\mathbf{R}_{0}}
$$

so that, if $\left\langle\mathbf{F}_{e}\right\rangle_{g}=0$ we will have $\mathbf{R}_{t}=\mathbf{R}_{0}$ at all time slices and

$$
\overline{\mathbf{R}}(t)=\int \mathbf{R} G_{g}\left(\mathbf{R}, \mathbf{R}_{0} ; t-t_{0}\right) \rho_{b 0}\left(\mathbf{R}_{0}\right) d \mathbf{R} d \mathbf{R}_{0}
$$

We also immediately see that the persistence will be the more important the steeper the external potential approaches its minimum. On an unstable equilibrium point of the potential (a maximum) the Green's function will be swallowed and eventually vanish in time.

Another interesting question is to determine the influence on the evolution of a spatially dependent diffusion coefficient $D(\mathbf{R})=v_{T}^{2} / \xi(\mathbf{R})$ rather than a constant. From Eq. (51) we see that around an equilibrium point for the external potential we will still have

$$
\mathbf{R}_{\tau} \simeq \mathbf{R}_{0}+\left.\tau\langle\nabla D\rangle_{g}\right|_{\mathbf{R}=\mathbf{R}_{0}}
$$

We will call this the "cage effect" of the solvent on the evolution of the solute particle.

In addition to the empty space boundary conditions other kinds are possible. Calling $\mathcal{I} \equiv D\left(\boldsymbol{\nabla}-\beta \mathbf{F}_{e}\right)$ the flux operator and $\partial \Omega$ the boundary of the space $\Omega$ where the solute particle is confined, the most general case are the radiation boundary conditions, namely

$$
\hat{\mathbf{n}}(\mathbf{R}) \cdot \mathcal{I}(\mathbf{R}) G\left(\mathbf{R}, \mathbf{R}_{0} ; t-t_{0}\right)=\omega G\left(\mathbf{R}, \mathbf{R}_{0} ; t-t_{0}\right), \quad \mathbf{R} \in \partial \Omega
$$

where $\hat{\mathbf{n}}(\mathbf{R})$ denotes a unit vector normal to the surface $\partial \Omega$. The reactivity is measured by the $\omega$ parameter. For $\omega=0$ we have a non-reactive or reflective boundary condition and for $\omega \rightarrow \infty$ we have a reaction boundary condition. So for a confined case we have to choose the required boundary condition and then determine the proper small time step Green's 
function $G_{g}$. For example for the solution in half space $X \geq 0$ with a reaction boundary condition at $X=0, G\left((0, Y, Z), \mathbf{R}_{0} ; t-t_{0}\right)=0$, we may use the image technique as follow

$$
\begin{aligned}
G_{g}\left(\mathbf{R}, \mathbf{R}_{0} ; \tau\right)= & \frac{e^{-\left[\left(X-X_{t}\right)^{2}+\left(Y-Y_{t}\right)^{2}+\left(Z-Z_{t}\right)^{2}\right] /\left.4\left\langle D_{i i}(\mathbf{R})\right\rangle_{g}\right|_{\mathbf{R}=\mathbf{R}_{0} \tau}}}{{\sqrt{4 \pi\left\langle D_{i i}(\mathbf{R})\right\rangle_{g} \mid \mathbf{R}_{\mathbf{R}} \mathbf{R}_{0} t}}^{3 N}}- \\
& \frac{e^{-\left[\left(X+X_{t}\right)^{2}+\left(Y-Y_{t}\right)^{2}+\left(Z-Z_{t}\right)^{2}\right] /\left.4\left\langle D_{i i}(\mathbf{R})\right\rangle_{g}\right|_{\mathbf{R}=\mathbf{R}_{0} \tau}}}{{\sqrt{4 \pi\left\langle D_{i i}(\mathbf{R})\right\rangle_{g} \mid \mathbf{R}=\mathbf{R}_{0} \tau}}^{3 N}} .
\end{aligned}
$$

\section{CONCLUSIONS}

In conclusion, for a colloidal suspension made up of one solute big and heavy particle and $N$ solvent small and light particles it is possible to reduce the Liouville dynamics to the Fokker-Planck dynamics by integrating over the coordinates and momenta of the solvent particles. Integrating furthermore on the momenta of the solute particle it is possible to reduce the Fokker-Planck equation to the Smoluchowski equation in the high friction regime. The two successive reductions are based each on a multiple time scale perturbation expansions. The first reduction was discovered in the second half of last century and the second reduction at the end of last century.

A simple Dynamical Monte Carlo (DynMC) algorithm is presented to solve the Smoluchowski equation for the Brownian motion of the solute particle in empty space and a thought computer experiment illustrated. We discuss how a steep external potential around its minimum will drive the approach to equilibrium more rapidly than the natural time scale $\tau_{b}$ and the cage effect due to the solvent. In particular we present a DynMC simulation on the harmonic case, with a constant diffusion, which well reproduces the exact analytical solution of the Smoluchowski equation. The simplicity of the simulation makes it very useful for the treatment of problems which do not have an analytic solution. We presented results for the average position of the solute particle as a function of time, but other quntities can be computed as for example the full Green's function starting from Eq. (57).

We discuss the proper boundary conditions to be imposed on the Smoluchowski second order partial differential equation in order to have a radiation, reflective, or reaction boundary and we give the small time expression for the Green's function in half space with a reaction boundary condition on the plane of separation using the image technique.

An interesting progress that could be made at the level of the Fokker-Planck description is to derive the expression for the friction coefficient for a colloidal patchy sticky hard sphere 
in a solvent of isotropic sticky hard spheres, in the spirit of Refs. [20 36] or in a solvent of penetrable square well particles, in the spirit of Refs. [37-42] or fluid mixtures adsorbed in porous disordered materials, as in Ref. [43, 44].

We leave as an open problem the generalization of the present treatment to the case of a classical Brownian solute macromulecule in a quantum solvent.

\section{Appendix A: Hermiticity of the Smoluchowski operator}

We will work in empty space. Then we introduce the Smoluchowski operator $\mathcal{L}_{S}(\mathbf{R})=$ $\nabla \mathbf{D} \rho_{0}(\mathbf{R}) \nabla \rho_{0}^{-1}(\mathbf{R})$. For any two functions $g(\mathbf{R})$ and $h(\mathbf{R})$ decaying to zero at spatial infinity faster than any inverse power we can write, using two times an integration by parts,

$$
\begin{aligned}
& \int d \mathbf{R} \frac{1}{\sqrt{\rho_{0}(\mathbf{R})}} g(\mathbf{R}) \mathcal{L}_{S}(\mathbf{R}) \sqrt{\rho_{0}(\mathbf{R})} h(\mathbf{R})= \\
& -\int d \mathbf{R} \rho_{0}(\mathbf{R}) \nabla\left(\frac{1}{\sqrt{\rho_{0}(\mathbf{R})}} g(\mathbf{R})\right) \mathbf{D} \nabla \frac{1}{\sqrt{\rho_{0}(\mathbf{R})}} h(\mathbf{R})= \\
& \int d \mathbf{R} \frac{1}{\sqrt{\rho_{0}(\mathbf{R})}} h(\mathbf{R}) \mathcal{L}_{S}(\mathbf{R}) \sqrt{\rho_{0}(\mathbf{R})} g(\mathbf{R}),
\end{aligned}
$$

which proves the Hermiticity, $\left[{\sqrt{\rho_{0}}}^{-1} \mathcal{L}_{S}{\sqrt{\rho_{0}}}^{\dagger}={\sqrt{\rho_{0}}}^{-1} \mathcal{L}_{S} \sqrt{\rho_{0}}\right.$, of the Smoluchowski operator. So that, from the Chapman-Kolmogorov relation

$$
G\left(\mathbf{R}_{0} \rightarrow \mathbf{R} ; t\right)=\int d \mathbf{R}_{1} G\left(\mathbf{R}_{1} \rightarrow \mathbf{R} ; \tau\right) G\left(\mathbf{R}_{0} \rightarrow \mathbf{R}_{1} ; t-\tau\right),
$$

follows

$$
\begin{aligned}
\frac{\partial}{\partial t} G\left(\mathbf{R}_{0} \rightarrow \mathbf{R} ; t\right) & =\mathcal{L}_{S}(\mathbf{R}) G\left(\mathbf{R}_{0} \rightarrow \mathbf{R} ; t\right) \\
& =\int d \mathbf{R}_{1} G\left(\mathbf{R}_{1} \rightarrow \mathbf{R} ; \tau\right) \mathcal{L}_{S}\left(\mathbf{R}_{1}\right) G\left(\mathbf{R}_{0} \rightarrow \mathbf{R}_{1} ; t-\tau\right) \\
& =\int d \mathbf{R}_{1} \frac{G\left(\mathbf{R}_{0} \rightarrow \mathbf{R}_{1} ; t-\tau\right)}{\rho_{0}\left(\mathbf{R}_{1}\right)} \mathcal{L}_{S}\left(\mathbf{R}_{1}\right) \rho_{0}\left(\mathbf{R}_{1}\right) G\left(\mathbf{R}_{1} \rightarrow \mathbf{R} ; \tau\right) \\
& \stackrel{\tau \rightarrow t}{\longrightarrow} \frac{1}{\rho_{0}\left(\mathbf{R}_{0}\right)} \mathcal{L}_{S}\left(\mathbf{R}_{0}\right) \rho_{0}\left(\mathbf{R}_{0}\right) G\left(\mathbf{R}_{0} \rightarrow \mathbf{R} ; t\right),
\end{aligned}
$$

where in the last limit we used the initial condition $G\left(\mathbf{R}_{0} \rightarrow \mathbf{R}_{1} ; 0\right)=\delta\left(\mathbf{R}_{0}-\mathbf{R}_{1}\right)$. And, using the symmetry of the left hand side of Eq. (A3) respect to exchange of $\mathbf{R}_{0} \leftrightarrow \mathbf{R}$, we finally find the detailed balance relation $\rho_{0}\left(\mathbf{R}_{0}\right) G\left(\mathbf{R}_{0} \rightarrow \mathbf{R} ; t\right)=\rho_{0}(\mathbf{R}) G\left(\mathbf{R} \rightarrow \mathbf{R}_{0} ; t\right)$.

[1] R. I. Cukier and J. M. Deutch, Phys. Rev. 177, 240 (1969). 
[2] O. Klein, Ark. Mat. Astron. Fys. 16, 1 (1921).

[3] H. A. Kramers, Physica 7, 284 (1940).

[4] L. Bocquet, Am. J. Phys. 65, 140 (1997).

[5] R. C. Balescu, Equilibrium and Non-Equilibrium Statistical Mechanics (Wiley, 1975).

[6] G. F. Mazenko, Nonequilibrium Statistical Mechanics (Wiley, 2008).

[7] L. E. Reichl, A Modern Course in Statistical Physics (Wiley, 2009).

[8] U. M. B. Marconi and S. Melchionna, J. Chem. Phys. 126, 184109 (2007).

[9] C. Wang, W. Yang, and S. Fu, in Colloidal Polymers: Synthesis and characterization, edited by A. Elaissari (Marcel Dekker Inc., 2003).

[10] K. Schulten and M. Tesch, Chem. Phys. 158, 421 (1991).

[11] D. Xu and K. Schulten, Chem. Phys. 182, 91 (1992).

[12] J.-P. Hansen and I. R. McDonald, Theory of Simple Liquids: With Applications to Soft Matter, 4th ed. (Academic Press, 2013).

[13] M. von Smoluchowski, Ann. Phys. 48, 1103 (1916).

[14] C. W. Gardiner, Handbook of Stochastic Methods (Springer, New York, 1983).

[15] M. H. Kalos and P. A. Whitlock, Monte Carlo Methods (John Wiley \& Sons, 1986).

[16] N. Metropolis, A. Rosenbluth, M. Rosenbluth, A. H. Teller, and E. Teller, J. Chem. Phys. 21, 1087 (1953).

[17] R. Kubo, Adv. Chem. Phys. 15, 101 (1969).

[18] S. Xiao-Jun, L. Xiao-Xia, Y. Yu-Liang, D. Jun-Feng, and Z. Jing-Shang, Commun. Theor. Phys. (Beijing China) 43, 1099 (2005).

[19] S. M. Klein, V. N. Manoharan, D. J. Pine, and F. F. Lange, Colloid Polym. Sci. 282, 7 (2003).

[20] R. Fantoni, D. Gazzillo, and A. Giacometti, J. Chem. Phys. 122, 034901 (2005).

[21] R. Fantoni, D. Gazzillo, and A. Giacometti, Phys. Rev. E 72, 011503 (2005).

[22] D. Gazzillo, A. Giacometti, R. Fantoni, and P. Sollich, Phys. Rev. E 74, 051407 (2006).

[23] D. Gazzillo, R. Fantoni, and A. Giacometti, Mol. Phys. 104, 3451 (2006).

[24] R. Fantoni, D. Gazzillo, A. Giacometti, and P. Sollich, J. Chem. Phys. 125, 164504 (2006).

[25] R. Fantoni, D. Gazzillo, A. Giacometti, M. A. Miller, and G. Pastore, J. Chem. Phys. 127, 234507 (2007).

[26] D. Gazzillo, R. Fantoni, and A. Giacometti, Phys. Rev. E 78, 021201 (2008). 
[27] D. Gazzillo, R. Fantoni, and A. Giacometti, Phys. Rev. E 80, 061207 (2009).

[28] R. Fantoni, A. Giacometti, F. Sciortino, and G. Pastore, Soft Matter 7, 2419 (2011).

[29] R. Fantoni, Eur. Phys. J. B 85, 108 (2012).

[30] R. Fantoni, J. W. O. Salari, and B. Klumperman, Phys. Rev. E 85, 061404 (2012).

[31] M. A. G. Maestre, R. Fantoni, A. Giacometti, and A. Santos, J. Chem. Phys. 138, 094904 (2013).

[32] R. Fantoni and G. Pastore, Europhys. Lett. 101, 46003 (2013).

[33] R. Fantoni and G. Pastore, Phys. Rev. E 87, 052303 (2013).

[34] R. Fantoni, A. Giacometti, M. A. G. Maestre, and A. Santos, J. Chem. Phys. 139, 174902 (2013).

[35] R. Fantoni, A. Giacometti, and A. Santos, J. Chem. Phys. 142, 224905 (2015).

[36] R. Fantoni and G. Pastore, Mol. Phys. 113, 2593 (2015).

[37] A. Santos, R. Fantoni, and A. Giacometti, Phys. Rev. E 77, 051206 (2008).

[38] R. Fantoni, A. Giacometti, A. Malijevský, and A. Santos, J. Chem. Phys. 131, 124106 (2009).

[39] R. Fantoni, A. Giacometti, A. Malijevský, and A. Santos, J. Chem. Phys. 133, 024101 (2010).

[40] R. Fantoni, J. Stat. Mech. , P07030 (2010).

[41] R. Fantoni, A. Malijevský, A. Santos, and A. Giacometti, Europhys. Lett. 93, 26002 (2011).

[42] R. Fantoni, A. Malijevský, A. Santos, and A. Giacometti, Mol. Phys. 109, 2723 (2011).

[43] P. G. D. S. Lucentini and G. Pellicane, Phys. Rev. Lett. 101, 246101 (2008).

[44] G. Pellicane, R. L. C. Vink, C. Caccamo, and H. Löwen, J. Phys.: Condens. Matter 20, 115101 (2008). 
This figure "Graphical-Abstract.jpg" is available in "jpg" format from: http://arxiv.org/ps/1809.08395v1 